\section{Modelo para avaliação da gestão de recursos humanos em saúde}

\author{
Evaluation model for human resource \\ management in health
}

\author{
1 Programa de Pós- \\ graduação em Saúde \\ Coletiva, Universidade \\ Federal de Santa Catarina, \\ Florianópolis, Brasil. \\ Correspondência \\ J. T. Lacerda \\ Programa de Pós-graduação \\ em Saúde Coletiva \\ Universidade Federal de Santa \\ Catarina. \\ Campus Universitário \\ Trindade, Florianópolis, SC \\ 88090-400, Brasil. \\ jtelino@gmail.com \\ jtelino@ccs.ufsc.br
}


tisfação no ambiente de trabalho e dificuldades no atendimento ao usuário em razão de interrupções nos serviços.

As políticas de recursos humanos devem contemplar ações de formação e desenvolvimento dos profissionais e facilitar a gestão do trabalho. As conferências nacionais de saúde e as conferências específicas de recursos humanos, que acontecem na saúde desde 1941, detectaram muitos problemas da área, mas muitas deliberações destes fóruns ainda não se efetivaram.

A partir da criação da Secretaria de Gestão do Trabalho e da Educação na Saúde (SGTES), com os Departamentos de Gestão da Educação e de Regulação do Trabalho, em 2003, o Ministério da Saúde assumiu com mais clareza seu papel, atuando na formulação e execução das políticas de Recursos Humanos para o Sistema Único de Saúde (SUS) 6.

Iniciativas importantes foram desencadeadas nos últimos anos, tais como a Política Nacional de Educação Permanente; o Pró-Saúde; a Mesa Nacional de Negociação Permanente do SUS; as Diretrizes Nacionais para a elaboração dos Planos de Carreira, Cargos e Salários dos trabalhadores do SUS; a Rede Nacional de Saúde do Trabalhador; a elaboração da Norma Operacional Básica de Recursos Humanos (NOB/RH-SUS) 7,8,9.

Nesse cenário, conhecer o desempenho das políticas e ações de recursos humanos é fundamental para identificação dos problemas, para o planejamento e o acompanhamento da área. Esse conhecimento pode ser gerado por meio de mecanismos de avaliação.

A avaliação consiste em fazer julgamento de valor a respeito de intervenções ou de seus componentes, para subsidiar a tomada de decisões, por meio de dispositivo que possa fornecer informações válidas e legítimas ${ }^{10}$. A necessidade de maximizar o uso dos recursos aplicados nos programas sociais fez com que a avaliação ganhasse importância na gestão pública. Os economistas iniciaram a utilização de métodos de análises de custos para os programas públicos, servindo como instrumento de gestão capaz de aperfeiçoar a ação governamental 11. Com a necessidade de fortalecimento da democracia, a avaliação pode ser utilizada como mecanismo de responsabilização do gestor perante terceiros 12 .

A gestão de recursos humanos é composta por um conjunto de ações que envolvem a contratação dos trabalhadores, a capacitação, a avaliação, a remuneração e o desenvolvimento de um ambiente de trabalho adequado ${ }^{13}$. Tomar decisões nessa área implica modificar determinadas realidades, processos, estruturas de tra- balhos, direitos e deveres, afetando a vida dos trabalhadores.

Conforme Silva \& Formigli 14, a avaliação poderia ser utilizada como prática sistemática na saúde para propiciar aos gestores as informações necessárias para a intervenção, e muitas informações registradas nos serviços acabam não sendo utilizadas para a tomada de decisões. Essas informações poderiam constituir-se em uma base importante para o monitoramento contínuo das ações de saúde.

Nos últimos anos, observou-se no país o delineamento de uma política de institucionalização da avaliação da atenção básica com o objetivo de transformar a avaliação em uma estratégia no cotidiano dos gestores e profissionais. A institucionalização da avaliação está associada ao desafio de consolidação do SUS por meio da qualificação das práticas, possibilitando maior resolutividade na atenção básica 15 .

Diante desse cenário, desenvolveu-se uma proposta de modelo de avaliação da gestão em recursos humanos na saúde, aplicado à atenção básica. Esse modelo poderá servir como instrumento para visualização da aplicação das políticas nessa área, subsidiando a tomada de decisões nos diferentes níveis de gestão da saúde.

\section{Metodologia}

O estudo desenvolvido consiste em uma pesquisa metodológica direcionada à descoberta de instrumentos de captação, coleta de dados e intervenção em uma determinada realidade. A base conceitual do modelo desenvolvido fundamentou-se na classificação de avaliação para a gestão apresentada por Novaes 16 .

O modelo teórico foi orientado pelo documento Princípios e Diretrizes para a Gestão do Trabalho no SUS (NOB/RH-SUS) ${ }^{1}$, que orienta a operacionalização de políticas e ações de recursos humanos no SUS nas três esferas de gestão. Está dividido em quatro grupos assumidos como dimensões na matriz avaliativa: Gestão do Trabalho no SUS, Desenvolvimento do Trabalhador do SUS, Saúde do Trabalhador do SUS, e Controle Social da Gestão do Trabalho no SUS. Nessa perspectiva, o modelo tem a função de aferir se a gestão municipal cumpre o previsto como função institucional no que se refere aos recursos humanos em saúde.

Os indicadores e medidas alocados nas dimensões foram elaborados em oficinas de consenso com um grupo de especialistas das áreas de avaliação e gestão, incluindo técnicos da Secretaria de Estado da Saúde de Santa Catarina que atuam na Atenção Básica, Saúde do Traba- 
lhador e Educação Permanente. O objetivo das oficinas foi envolver os profissionais do serviço no processo de avaliação, aproximando a proposta às necessidades do SUS. A etapa de identificação dos indicadores teve caráter qualitativo e envolveu a discussão das questões mais relevantes da gestão de recursos humanos em cada uma das áreas, sendo as decisões tomadas por consenso.

Os dados para a composição dos indicadores foram coletados de duas formas: (a) diretamente nos municípios por meio de formulário elaborado e testado previamente; (b) em documentos e relatórios da comissão organizadora da Conferência Estadual de Saúde e nas listas de presença do Pólo Litoral Centro.

A amostra utilizada para testar a aplicabilidade do modelo foi composta por todos os municípios da macrorregião da Grande Florianópolis, Estado de Santa Catarina, Brasil, num total de 22 unidades, sendo que, destes, 15 disponibilizaram todas as informações. Os objetivos do estudo foram apresentados aos gestores municipais em reunião do Colegiado de Gestão Regional, momento em que apresentavam concordância e agendavam horário de visita para coleta. Os formulários foram preenchidos pelos responsáveis e técnicos da área, com orientação pessoal ou por telefone, e conferidos pela pesquisadora no momento da entrega.

A análise dos dados foi realizada de forma percentual e os parâmetros dos indicadores foram definidos com base nas recomendações encontradas na literatura e nas normas vigentes. Os dados utilizados no estudo são de domínio público, não exigindo autorização do Comitê de Ética em Pesquisa.

\section{Resultados}

\section{O modelo proposto}

O modelo proposto permitiu analisar o subsistema "Gestão de Recursos Humanos" de forma integrada, com uma imagem detalhada do desempenho dos municípios em relação aos trabalhadores do SUS. A matriz avaliativa resume a representação teórica do objeto avaliado, resultante das oficinas de consenso e subsidiadas pela literatura e legislação vigente (Tabela 1).

Na dimensão Gestão do Trabalho, com três indicadores, a "qualificação do vínculo" incorpora a idéia de que o trabalhador que possui inserção fixa no sistema tem melhores garantias trabalhistas e está menos sujeito à precarização do trabalho. Foram excluídos da pesquisa os agentes comunitários de saúde, dada sua recente inserção nos quadros e as indefinições quanto à melhor forma de vinculação destes trabalhadores. Assumiu-se que os municípios com pelo menos $94 \%$ de trabalhadores estatutários estejam em situação "adequada", considerando-se que em torno de $6 \%$ dos cargos normalmente são comissionados, conforme o estudo de Arias et al. 17 . A "fixação dos trabalhadores no município" fundamentou-se na constatação de que altas taxas de rotatividade prejudicam o funcionamento dos serviços e geram custos desnecessários. Mudanças constantes de profissionais podem gerar interrupção nas atividades e dificultar o vínculo dos profissionais com os pacientes e a comunidade. Significa que a gestão não está conseguindo equilibrar as necessidades e expectativas dos profissionais com as dos serviços. Para aferir esse indicador utilizou-se o total de médicos desligados no período de 2005 a 2006 em relação à média de médicos existentes neste mesmo período. A definição do parâmetro adequado seguiu o estudo de Campos \& Malik 4. A "valorização do trabalhador" representa um dos fatores determinantes para a motivação e manutenção do trabalhador nos serviços. A análise considerou como "boa valorização" a gestão que possuía Plano de Carreira, Cargos e Salários (PCCS) implantado.

Na dimensão Desenvolvimento do Trabalhador do SUS, o indicador "empenho da gestão na qualificação profissional" foi medido por meio da capacitação dos médicos durante o ano de 2006. Essa definição considerou a dificuldade em obter a participação da categoria médica em cursos promovidos pelo sistema de saúde. Pressupôsse que a gestão que realiza capacitações com a participação do médico esteja conseguindo o mesmo para os demais profissionais. Tomou-se como padrão que todo médico participe de pelo menos uma capacitação durante o ano. A "realização da Educação Permanente em serviço" considerou as oportunidades previstas ao desenvolvimento das equipes, e que as reuniões mensais de planejamento são ocasiões propiciadoras da educação em serviço e de planejamento e desenvolvimento do trabalho de forma coletiva. Todas as Equipes de Saúde da Família deveriam contar com essa oportunidade. Na "inserção do município nas decisões de educação permanente da região" considerou-se a vinculação do município a um Pólo de Educação Permanente e sua participação efetiva nas reuniões. Nas atividades de articulação do Pólo é importante haver o máximo de freqüência para que os representantes acompanhem o raciocínio das discussões e participem das decisões de forma produtiva. A freqüência mínima de $75 \%$ nas reuniões do Pólo foi estabelecida com base nos padrões da área da educação. 
Tabela 1

Matriz de avaliação: dimensões, indicadores, medidas e parâmetros de análise.

\begin{tabular}{|c|c|c|c|}
\hline Dimensões & Indicadores & Medidas & Parâmetros \\
\hline \multirow[t]{6}{*}{ Gestão do trabalho no SUS } & Qualificação do vínculo de trabalho & & $<94 \%$ : Inadequado \\
\hline & & $\begin{array}{l}\text { com vínculo estatutário em } 2005 \text { e } \\
2006\end{array}$ & $\geq 94 \%:$ Adequado \\
\hline & Fixação do trabalhador no município & Índice de rotatividade na função de & 0-25\%: Bom \\
\hline & & médico em 2005 e 2006 & $>25 \%:$ Ruim \\
\hline & Valorização do trabalhador & Existência de Plano de Carreira, & Sim: Bom \\
\hline & & $\begin{array}{l}\text { Cargos e Salários em funcionamento } \\
\text { em } 2006\end{array}$ & Não: Ruim \\
\hline \multirow{5}{*}{$\begin{array}{l}\text { Desenvolvimento do } \\
\text { Trabalhador no SUS }\end{array}$} & Empenho da gestão na qualificação & Percentual de médicos que partici- & 100\%: Bom \\
\hline & profissional & param de capacitações em 2006 & < 100\%: Ruim \\
\hline & Realização de Educação Permanente & Percentual de Equipes de Saúde da & 100\%: Bom \\
\hline & em serviço & $\begin{array}{c}\text { Família que realizaram reuniões de } \\
\text { planejamento em } 2006\end{array}$ & $<$ 100\%: Ruim \\
\hline & $\begin{array}{l}\text { Inserção do município nas decisões } \\
\text { sobre Educação Permanente }\end{array}$ & $\begin{array}{c}\text { Percentual de presenças nas reuniões } \\
\text { dos Pólos de Educação Permanente } \\
\text { em } 2005 \text { e } 2006\end{array}$ & $\begin{array}{l}<75 \% \text { : Insatisfatória } \\
\geq 75 \% \text { : Satisfatória }\end{array}$ \\
\hline \multirow[t]{7}{*}{$\begin{array}{l}\text { Saúde do Trabalhador } \\
\text { do SUS }\end{array}$} & $\begin{array}{l}\text { Impacto das ações de prevenção e } \\
\text { controle de riscos ocupacionais }\end{array}$ & $\begin{array}{l}\text { Percentual de acidentes de trabalho } \\
\qquad \text { em } 2006\end{array}$ & $\begin{array}{l}\text { 0: Bom } \\
\text { > 0: Ruim }\end{array}$ \\
\hline & Adequação do ambiente psicológico & Percentual de afastamentos por & 0: Bom \\
\hline & de trabalho & depressão em 2006 & > 0: Ruim \\
\hline & Assistência à saúde dos trabalha- & Acesso a serviços de Comissões & Sim: Bom \\
\hline & dores & Locais de Saúde do Trabalhador ou & Não: Ruim \\
\hline & & Centros de Referência em Saúde do & \\
\hline & & Trabalhador em 2006 & \\
\hline \multirow{5}{*}{$\begin{array}{l}\text { Controle Social da Gestão } \\
\text { de RH no SUS }\end{array}$} & Organização do controle social para & Existência de Comissões Intersetori- & Sim: Bom \\
\hline & atuar nas questões de RH & $\begin{array}{c}\text { ais de RH, Câmaras Técnicas de RH, } \\
\text { ou outra, nos Conselhos Municipais } \\
\text { de Saúde em } 2006\end{array}$ & Não: Ruim \\
\hline & Participação dos Conselhos Munici- & Existência de discussões sobre temas & Sim: Bom \\
\hline & pais de Saúde nos problemas de RH & $\begin{array}{c}\text { de RH nos Conselhos Municipais de } \\
\text { Saúde em } 2006\end{array}$ & Não: Ruim \\
\hline & $\begin{array}{c}\text { Articulação com a sociedade na } \\
\text { definição de políticas de RH }\end{array}$ & $\begin{array}{c}\text { Existência de deliberações sobre } \\
\text { temas de RH nas Conferências Mu- } \\
\text { nicipais de Saúde em } 2007\end{array}$ & $\begin{array}{l}\text { Sim: Bom } \\
\text { Não: Ruim }\end{array}$ \\
\hline
\end{tabular}

RH: Recursos Humanos; SUS: Sistema Único de Saúde.

Na dimensão Saúde do Trabalhador do SUS, o indicador "impacto de ações de prevenção e controle de riscos ocupacionais" considerou a responsabilidade do gestor na prevenção e controle de riscos no ambiente de trabalho. Uma boa gestão deve ter $0 \%$ de afastamentos por acidentes de trabalho. No indicador "adequação do ambiente psicológico de trabalho" está a idéia de que baixos índices de afastamentos por depressão podem indicar a existência de preocupação por parte da gestão com ações de manutenção da saúde dos trabalhadores, ou pelo menos com a existência de um bom ambiente de trabalho.
Nenhum afastamento por depressão foi o parâmetro de boa adequação. A "assistência dos trabalhadores" pode ser viabilizada por meio de ações realizadas diretamente pelo município, não deixando apenas para o trabalhador a responsabilidade de buscar atendimento quando necessário. Para esse indicador, adotou-se como parâmetro o acesso a Comissões Locais de Saúde do Trabalhador ou aos Centros de Referência em Saúde do Trabalhador.

A dimensão Controle Social da Gestão de Recursos Humanos no SUS procurou medir a relação da sociedade com o SUS nas questões que 
envolvem a gestão dos trabalhadores. Segundo a NOB/RH-SUS, devem ser instituídas Comissões Intersetoriais de Recursos Humanos (CIRH) nos Conselhos de Saúde. Essas comissões têm a responsabilidade de acompanhar a implementação das deliberações dos conselhos sobre a gestão de RH no SUS, na respectiva instância de gestão. Os conselhos também podem instalar Câmaras Técnicas que analisam preliminarmente as matérias para que possam ser deliberadas pelo conselho facilitando a discussão. Essa idéia é representada pelo indicador "organização do controle social para definições e acompanhamento das deliberações sobre RH no município", medida pela existência de CIRH, Câmaras Técnicas de Recursos Humanos ou outro tipo de comissão de RH nos Conselhos Municipais de Saúde, no ano de 2006. Optou-se por considerar como "boa organização" a existência de uma ou outra comissão. Para a análise do indicador "participação dos Conselhos Municipais de Saúde nos problemas de RH" considerou-se como positivo quando o conselho discutiu temas de RH em suas reuniões. A existência de discussões sobre problemas de RH nos conselhos demonstra sensibilidade da comunidade na questão do trabalho e no controle sobre a ação do Estado, favorecendo a transparência e a democracia nas decisões. $\mathrm{O}$ indicador "articulação do município com a sociedade para a definição de políticas de RH” foi avaliado considerando-se a existência de propostas de $\mathrm{RH}$ na última Conferência Municipal de Saúde de cada município. Essa decisão fundamentou-se na orientação da NOB/RH-SUS já mencionada, e no entendimento que as conferências de saúde representam espaços importantes para a discussão de propostas que fundamentam a construção democrática das políticas de saúde. Considerouse como "boa articulação" a existência de pelo menos uma proposta sobre RH (Gestão do Trabalho, Desenvolvimento do Trabalhador e Saúde do Trabalhador) aprovada.

Ainda com base na revisão da literatura, normas e legislação, foi proposta uma matriz avaliativa para emissão de juízo de valor (Figura 1).

\section{O teste de aplicabilidade}

Participaram da pesquisa 15 dos 22 municípios da macrorregião da Grande Florianópolis. A exclusão de sete municípios foi ocasionada pela não disponibilidade de dados no período estudado e pela dificuldade em obter informações gerenciadas fora da Secretaria Municipal de Saúde, tais como número e vínculo de profissionais. Essas dificuldades apontam para a falta de mecanismos de registro de dados de recursos humanos e um provável desconforto da gestão em revelar a sua realidade quanto a questões trabalhistas.

O modelo proposto demonstrou viabilidade de aplicação para municípios de diferentes portes populacionais, e o conjunto de indicadores demonstrou ser adequado para a avaliação da gestão de recursos humanos na atenção básica. Os dados coletados permitiram gerar as informações necessárias, e não foram encontradas dificuldades na utilização do instrumento de coleta de dados. Os dados da Tabela 2 demonstram que o comportamento dos indicadores foi diferenciado entre os municípios, mas não há indicação de tendências relacionadas ao porte populacional.

De maneira geral os resultados são pouco animadores no seu conjunto. Os indicadores que apresentaram melhores desempenhos foram: "impacto de ações de prevenção e controle de riscos ocupacionais”, "adequação do ambiente psicológico de trabalho" e "realização de educação permanente em serviço". Os desempenhos mais baixos na maioria dos municípios referiram-se à "organização do controle social para atuar nas questões de RH”, "inserção dos municípios nas decisões de educação permanente da região” e "qualificação do vínculo de trabalho no SUS".

A Gestão do Trabalho no SUS apresentou pior desempenho no indicador "qualificação do vínculo de trabalho" - apenas um município teve pelo menos $94 \%$ de seus trabalhadores contratados em regime jurídico único e $47 \%$ dos municípios estudados possuem mais de $50 \%$ de trabalhadores estatutários. A "fixação dos trabalhadores de saúde" do município, medida pela taxa de rotatividade dos médicos, revelou-se um pouco melhor que a situação dos vínculos trabalhistas. Dentre os municípios estudados, $47 \%$ apresentaram taxas de rotatividade aceitáveis, ou seja, menor ou igual a $25 \%$. Desses, seis municípios, pequenos e médios, não apresentaram rotatividade. A rotatividade geral variou de 0 a 238\%. Para os 15 municípios avaliados verificouse a existência de alta rotatividade em mais de $50 \%$, indicando que a fixação dos profissionais é ruim nessa macrorregião. $\mathrm{Na}$ "valorização do trabalhador" identificou-se que apenas $40 \%$ dos municípios estudados possuíam PCCS implantado no ano de 2006.

No desenvolvimento do trabalhador do SUS dois indicadores apresentaram desempenho ruim no conjunto de municípios. O "empenho da gestão na qualificação profissional” e a "inserção dos municípios nas decisões sobre educação permanente" apresentaram-se adequados apenas em dois e um municípios, respectivamente. Para o primeiro, destaca-se que em $73 \%$ dos municípios nenhum médico participou de 

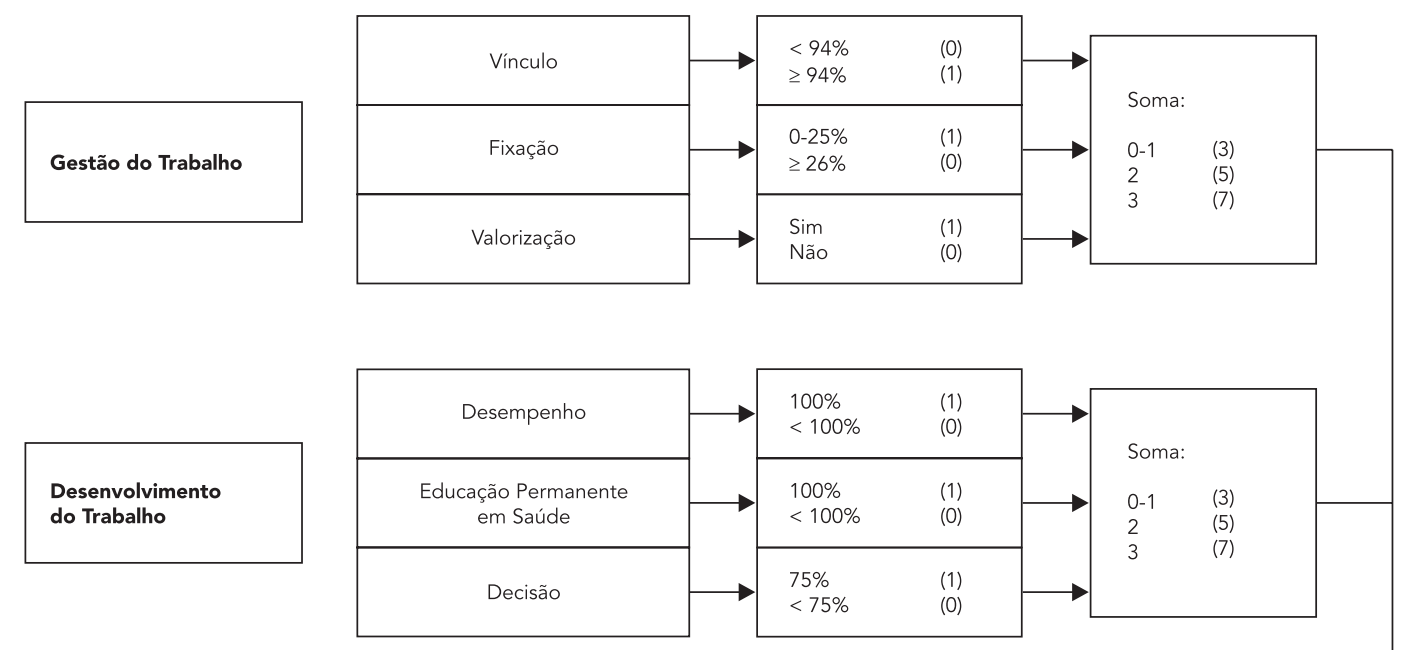

Classificação do Município
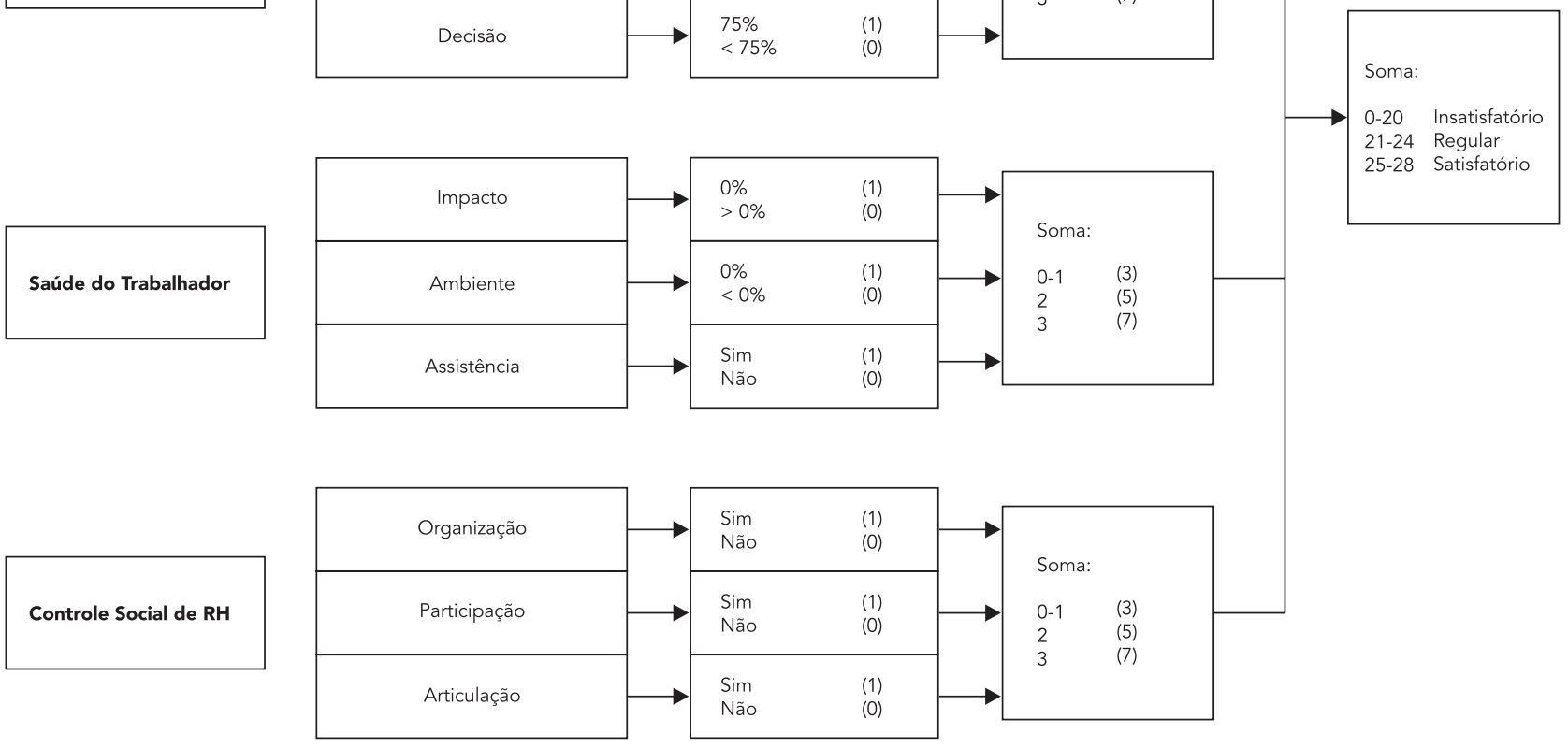

qualquer capacitação promovida ou articulada pelo município. A inserção nas decisões sobre educação permanente foi incipiente, com um município apresentando $78 \%$ de freqüência às reuniões e um com $50 \%$. O indicador de "realização da educação permanente em serviço" foi um dos que demonstrou o melhor desempenho. $\mathrm{Na}$ maioria dos municípios (60\%), todas as Equipes de Saúde da Família realizaram reuniões mensais de planejamento no ano de 2006. Os demais municípios informaram que menos da metade das Equipes de Saúde da Família realizaram reuniões mensais naquele ano.
Em Saúde do Trabalhador do SUS estão os dois indicadores que apresentaram os melhores resultados na avaliação. O indicador "impacto das ações de prevenção e controle de riscos ocupacionais" demonstrou que 90,3\% dos municípios estudados não apresentaram casos de afastamentos por acidentes de trabalho. Os demais $(6,7 \%)$ referem-se à resposta não informada, que foi considerada como negativa para a gestão. Na "adequação do ambiente psicológico de trabalho para o desenvolvimento das atividades" a maioria dos municípios $(66,7 \%)$ não relatou casos de depressão no período estudado. A ausência de 
Tabela 2

Resultados da avaliação da gestão de Recursos Humanos (RH) segundo dimensão e indicador, por município, na Macrorregional da Grande Florianópolis, Santa Catarina, Brasil.

\begin{tabular}{|c|c|c|c|c|c|c|c|c|c|c|c|c|c|c|c|}
\hline \multirow[t]{2}{*}{ Municípios } & \multirow[t]{2}{*}{ População } & \multicolumn{3}{|c|}{ Gestão do Trabalho } & \multicolumn{3}{|c|}{$\begin{array}{l}\text { Desenvolvimento do } \\
\text { Trabalhador }\end{array}$} & \multicolumn{3}{|c|}{ Saúde do Trabalhador } & \multicolumn{3}{|c|}{$\begin{array}{c}\text { Controle Social de } \\
\text { Gestão de RH }\end{array}$} & \multicolumn{2}{|c|}{ Resultados } \\
\hline & & G1 & G2 & G3 & D1 & D2 & D3 & S1 & S2 & S3 & $\mathrm{C} 1$ & $\mathrm{C} 2$ & C3 & Total & $\%$ \\
\hline 1 & 2.668 & & & & & & & & & & & & & 4 & 33,3 \\
\hline 2 & 2.841 & & & & & & & & & & & & & 4 & 33,3 \\
\hline 3 & 2.992 & & & & & & & & & & & & & 5 & 41,7 \\
\hline 4 & 3.101 & & & & & & & & & & & & & 5 & 41,7 \\
\hline 5 & 3.348 & & & & & & & & & & & & & 5 & 41,7 \\
\hline 6 & 3.868 & & & & & & & & & & & & & 3 & 25,0 \\
\hline 7 & 5.138 & & & & & & & & & & & & & 6 & 50,0 \\
\hline 8 & 5.410 & & & & & & & & & & & & & 6 & 50,0 \\
\hline 9 & 7.042 & & & & & & & & & & & & & 4 & 33,3 \\
\hline 10 & 13.050 & & & & & & & & & & & & & 2 & 16,7 \\
\hline 11 & 15.562 & & & & & & & & & & & & & 4 & 33,3 \\
\hline 12 & 16.410 & & & & & & & & & & & & & 3 & 25,0 \\
\hline 13 & 18.245 & & & & & & & & & & & & & 3 & 25,0 \\
\hline 14 & 58.433 & & & & & & & & & & & & & 2 & 16,7 \\
\hline 15 & 406.566 & & & & & & & & & & & & & 5 & 41,7 \\
\hline Total & & 1 & 7 & 6 & 2 & 9 & 1 & 14 & 10 & 2 & 0 & 6 & 3 & & \\
\hline$\%$ & & 6,7 & 46,7 & 40,0 & 13,3 & 60,0 & 6,7 & 93,3 & 66,7 & 13,3 & 0,0 & 40,0 & 20,0 & & \\
\hline
\end{tabular}

G1: Qualificação do vínculo de trabalho; G2: Fixação do trabalhador no município; G3: Valorização do trabalhador; D1: Empenho da gestão na qualificação profissional; D2: Realização de Educação Permanente em serviço; D3: Inserção do município nas decisões sobre Educação Permanente; S1: Impacto das ações de prevenção e controle de riscos ocupacionais; S2: Adequação do ambiente de trabalho; S3: Assistência à saúde dos trabalhadores; C1: Organização do controle social para atuar nas questões de RH; C2: Participação dos Conselhos Municipais de Saúde nos problemas de RH; C3: Articulação com a sociedade na definição de políticas de $\mathrm{RH}$.

Legenda: Parâmetro alcançado

Parâmetro não alcançado

casos de depressão sugere que na maioria dos municípios estudados existe um ambiente psicológico de trabalho propício para a realização das atividades. Os casos de depressão apontados ocorreram por mais de uma vez durante os anos de 2005 e 2006, podendo estar associadas com as questões relacionadas ao trabalho. Por outro lado, "a assistência à saúde dos trabalhadores", medida pelo acesso a Comissões Locais de Saúde do Trabalhador organizadas pelo município ou acesso a serviços de Centros de Referência de Saúde do Trabalhador na região, apresentou resultados ruins - apenas dois municípios estudados (13,3\%) relataram acesso a serviços de Centros de Referência e nenhum identificou a existência de Comissões Locais de Saúde do Trabalhador no município.

O Controle Social da Gestão de Recursos Humanos no SUS foi a dimensão com os piores resultados. A "organização do Controle Social” para atuar nas questões de recursos humanos foi inexistente na totalidade dos municípios. Em $100 \%$ dos municípios estudados não havia algum tipo de comissão para RH em funcionamento em 2006. Isso demonstra que os Conselhos Municipais de Saúde, de maneira geral, não se encontravam organizados para melhor discutir e fazer acontecer suas deliberações. Da mesma forma, na "participação do Controle Social nos problemas relacionados a RH", detectou-se que no ano de 2006 apenas 40\% dos municípios estudados discutiram assuntos ligados a RH. A "participação da sociedade na definição de políticas de recursos humanos" foi analisada com base na existência de definições sobre RH nas Conferências Municipais de Saúde. Como resultado, identificou-se que apenas $20 \%$ dos municípios apresentaram definições nessa área.

O modelo proposto avaliou o resultado de quatro dimensões, com 12 indicadores, identifi- 
cando seu comportamento apenas no conjunto de municípios estudados em razão do tamanho reduzido da amostra. Na Figura 1 propõe-se o complemento do modelo destinado à avaliação do desempenho dos municípios de forma individual e global, estabelecendo-se julgamento de valor por indicador e dimensão que pode ser aplicado em amostras maiores.

\section{Discussão}

A aplicação do modelo demonstrou-se viável e evidenciou a precariedade ou dificuldade de acesso aos sistemas de informação no que se refere aos recursos humanos em saúde. A preocupação em preservar o sigilo do servidor e a pulverização das informações de recursos humanos em diferentes subsistemas são limitações de gestão já reportadas por outros autores 18 . Todavia, modelos de avaliação que façam uso dessas informações constituem estímulo à organização racional desses dados para que possam subsidiar o planejamento e a decisão nos sistemas municipais de saúde.

Os indicadores utilizados na dimensão Gestão do Trabalho no SUS abordam questões sensíveis no sistema público. Admitir a ausência de PCCS e a existência de vínculos trabalhistas precários nos municípios não é uma posição confortável para os gestores, tanto quanto evidenciar a alta rotatividade dos profissionais da saúde. Arias et al. 17, quando estudaram os PCCS das Secretarias de Estado e dos Municípios, em 2004 , identificaram que apenas $38,5 \%$ das secretarias municipais de saúde possuíam PCCS implantado. Da mesma forma, Pierantoni et al. 19 detectaram apenas $20 \%$ de secretarias de saúde com PCCS específicos para os trabalhadores da saúde e outros $29 \%$ com PCCS geral para todos os funcionários. Os entrevistados reportaram inúmeras dificuldades - legais, financeiras, técnicas e políticas - como entraves para o estabelecimento de PCCS. O mesmo estudo detectou uma parcela reduzida de gestores que não contavam com trabalhadores desprotegidos em seus quadros. Pesquisa realizada por Borrelli 3 , com profissionais de saúde da zona rural de Sergipe, identificou taxas de rotatividade variadas ficando entre 0 a $300 \%$ nos municípios estudados, com uma média geral de 84,9\%, também considerada alta pela pesquisadora.

A valorização do trabalhador pode ser realizada por diferentes meios capazes de recompensar o trabalho realizado: recompensas financeiras diretas, como salário, prêmios, comissões; recompensas financeiras indiretas em forma de férias, gratificações, horas-extras, 13o salário, adicionais; recompensas não-financeiras oferecidas em forma de oportunidades de desenvolvimento, reconhecimento, segurança no emprego, qualidade de vida no trabalho, promoções, liberdade, autonomia 20. Os relatos da literatura e os resultados encontrados na aplicação do modelo demonstram a necessidade de analisar esse aspecto nos sistemas de saúde, haja vista que somente $40 \%$ dos municípios estudados relataram a presença de PCCS. Os resultados da aplicação do modelo são compatíveis com as indicações da literatura, sugerindo a validade dos indicadores propostos nessa dimensão, uma vez que foram capazes de constatar desempenhos ruins na amostra de municípios.

Na dimensão Desenvolvimento dos Trabalhadores do SUS, os indicadores conduzem a gestão à reflexão sobre suas atribuições na formação de seus profissionais. A lógica da educação permanente para o SUS enfatiza as necessidades locais e propõe a transformação das práticas profissionais por meio da reflexão crítica do processo de trabalho. Nessa perspectiva, os processos de capacitação do pessoal da saúde devem ser estruturados a partir da problematização do processo de trabalho, tomando como referencial as necessidades de saúde das pessoas, a gestão setorial e a promoção da saúde ${ }^{8}$. Alternativas simples, como as reuniões em serviço, podem contribuir na qualificação dos profissionais, na aproximação dos membros das equipes e na produção de soluções para a melhoria das ações e serviços de saúde. Realizar reuniões periódicas deveria fazer parte do cotidiano da coordenação das equipes, podendo ser organizadas sistematicamente sem implicar aumento de custos. O indicador proposto - reunião de planejamento, inserida como uma estratégia de educação permanente em serviço - apresentou bom desempenho na maioria dos municípios. Todavia, o indicador pode ser aprimorado, incluindo alguns aspectos das pautas e atas dessas reuniões para verificar se elas cumprem o seu papel na educação permanente dos trabalhadores ou apenas tratam de questões administrativas do serviço.

Os outros dois indicadores reportam a responsabilidade do município em oferecer cursos, aperfeiçoamentos e capacitações para o bom desempenho profissional de seus trabalhadores. Isso pode ocorrer por meio da realização de eventos por sua própria iniciativa ou participando ativamente das decisões sobre Educação Permanente na região, indicando e reivindicando suas necessidades de formação. Os sistemas municipais de saúde ainda não assumiram essa responsabilidade e os resultados da aplicação do modelo foram coerentes com essa realidade, demonstrando a existência de poucas oportuni- 
dades de capacitação e baixa participação dos municípios pesquisados na Política Nacional de Educação Permanente. O indicador "empenho da gestão" detectou a pequena capacidade que os municípios tiveram em mobilizar os profissionais para participação em atividades promovidas ou articuladas pelo município. As capacitações são indispensáveis para a motivação dos profissionais e para a manutenção da qualidade dos serviços. Cabe à gestão municipal promover capacitações para seus trabalhadores ou buscar parcerias para sua viabilização, por meio da Política Nacional de Educação Permanente 1,21. A participação do município nas discussões da política de educação permanente na região representa um espaço importante para articulação de capacitações para os trabalhadores com recursos do Ministério da Saúde e, atualmente, uma responsabilidade da gestão quanto à condução da política em âmbito local por intermédio dos Colegiados de Gestão Regional 21 .

Na dimensão Saúde do Trabalhador foram observados os melhores resultados, porém alguns fatores que demandam atenção. $\mathrm{O}$ indicador "impacto das ações de prevenção e controle de riscos ocupacionais" foi medido pelo percentual de acidentes de trabalho no ano, mas não detectar acidentes de trabalho pode estar associado ao não registro de acidentes de trabalho, comum em muitas instituições; também, os acidentes de trabalho podem ser evitados pela ação do trabalhador no cuidado de sua própria saúde. De qualquer forma, a falta de atenção da gestão em ações de prevenção aumenta as chances de riscos aos trabalhadores, reafirmando a importância da mensuração desse indicador. A leitura dos resultados deve ser realizada sob olhar crítico em cada realidade estudada. $\mathrm{Na}$ "adequação do ambiente psicológico de trabalho para o desenvolvimento das atividades" a medida utilizada foi o número de casos de depressão no período estudado. Embora a relação entre as causas da doença e o processo de trabalho não seja facilmente estabelecida, a ausência de casos de depressão tem potencial para demonstrar a existência de um ambiente psicológico de trabalho propício para a realização das atividades. Estudos têm demonstrado que os transtornos mentais e do comportamento estão entre as causas relevantes das doenças do trabalho nos órgãos públicos. Pesquisa desenvolvida em 2006 22, com os servidores públicos federais civis do poder executivo no Estado de Santa Catarina, revelou que os transtornos mentais e do comportamento representaram a segunda causa dentre as doenças relacionadas ao trabalho. Outro estudo ${ }^{23}$, sobre os problemas de saúde dos servidores públicos da Secretaria de Estado da Saúde e da Secretaria de Estado de Administração em Santa Catarina, no período de 1995 a 2005, demonstrou que os principais motivos dos afastamentos do período estavam relacionados aos transtornos mentais e comportamentais (25\%), tendo como maior freqüência os transtornos depressivos moderados e os episódios depressivos (32\% dos casos).

$\mathrm{O}$ indicador "assistência à saúde dos trabalhadores" evidenciou a ausência de estruturas de Comissões Locais de Saúde do Trabalhador e baixo acesso aos Centros de Referência de Saúde do Trabalhador. A ausência de estratégias para viabilizar o acesso dos trabalhadores à assistência por problemas originados no trabalho pode revelar uma baixa preocupação da gestão com a recuperação da saúde dos trabalhadores e um provável distanciamento dos municípios com relação às políticas nesta área. Os resultados da aplicação indicam a manutenção do indicador, uma vez que foi capaz de detectar evidências empíricas quanto ao cuidado com a saúde dos trabalhadores em saúde.

Na dimensão Controle Social da Gestão de Recursos Humanos no SUS estão os indicadores menos freqüentes nas análises deste tópico. As CIRH ou similares nos Conselhos Municipais de Saúde cumprem a função de facilitar a participação da comunidade na formulação de estratégias de operacionalização das políticas de saúde e no controle da sua execução. As propostas e estratégias de seleção, contratação, fixação, alocação e estímulo dos profissionais que atuam na saúde devem ser matéria de interesse dos Conselhos de Saúde e necessitam de discussões fundamentadas nos aspectos legais, técnicos e sociais para serem deliberados com responsabilidade 1 . Além dos Conselhos Municipais de Saúde, as Conferências de Saúde constituem espaço privilegiado de discussão da sociedade a respeito dos profissionais que atuam em saúde nos municípios. Cabe à gestão local estimular e propiciar esses espaços e a inserção dessa pauta nas comissões, reuniões, conselhos e conferências para garantir a legitimidade das políticas públicas de recursos humanos no município.

A aplicação do modelo indicou mais de 50\% dos municípios com desempenhos classificados como "ruim" em todos os indicadores nessa dimensão de análise. Foi constatada a inexistência de Comissões Intersetoriais em 100\% dos municípios, $40 \%$ discutiram assuntos ligados a $\mathrm{RH}$ e apenas $20 \%$ apresentaram definições de RH em Conferências Municipais de Saúde. Tais resultados sugerem que as discussões sobre RH nos Conselhos de Saúde, quando acontecem, são ocasionais e improvisadas. Frente à alta rotatividade de pessoal observada, considera-se pouco provável que no período estudado não houvesse 
demandas para discussões junto aos conselhos. A garantia de espaço para discussão colegiada e deliberativa favorece o diálogo, a contestação e a negociação democrática das necessidades e interesses de cada segmento participante usuários, prestadores de serviços, profissionais e governo 24. Logo, a realização de conferências municipais e a atuação dos conselhos nas questões de recursos humanos são indispensáveis para o avanço das políticas de recursos humanos no SUS. A importância da manutenção desses indicadores reside na possibilidade de alertarem os gestores sobre a necessidade de construir propostas democráticas para a gestão de recursos humanos, com a participação dos principais interessados na conformação de quadros funcionais qualificados e satisfeitos.

\section{Conclusão}

A proposta permitiu analisar o subsistema gestão de recursos humanos de forma integrada nos aspectos de gestão do trabalho, desenvolvimento do trabalhador, saúde do trabalhador e controle social da gestão de recursos humanos, trazendo uma imagem ampliada da área, propondo um instrumento para avaliação global das políticas vigentes.
O estudo apresentado teve como foco o desenvolvimento e aplicação de um modelo para avaliação da gestão de RH, não tendo como prioridade a avaliação do desempenho dos municípios pesquisados.

Por meio do teste de aplicabilidade verificouse que o modelo é viável na atenção básica, em municípios de diferentes tamanhos, uma vez que os resultados encontrados não apresentaram variações importantes em razão do porte populacional.

Os indicadores utilizados demonstraram validade para a avaliação da gestão de recursos humanos dentro das políticas vigentes. Os dados coletados foram capazes de gerar as informações necessárias à medição dos indicadores definidos.

Os sistemas de informação existentes na área de recursos humanos da saúde ainda não favorecem a geração de dados contínuos e sistemáticos que facilitem a institucionalização da avaliação e a realização de estudos ampliados. Todavia, a realização de estudos avaliativos nessa área é fundamental para a transformação dessa realidade.

\section{Resumo}

Este artigo apresenta um modelo de avaliação da gestão de recursos humanos na saúde. Trata-se de uma pesquisa metodológica, estruturada por meio de indicadores testados a partir de pesquisa avaliativa. Utilizou-se como base a Norma Operacional Básica de Recursos Humanos (NOB/RH-SUS), estabelecendo-se como dimensões: Gestão do Trabalho, Desenvolvimento do Trabalhador, Saúde do Trabalhador e Controle Social. O modelo foi discutido e aperfeiçoado em oficinas de consenso com especialistas em avaliação e gestão de recursos humanos, configurando uma matriz de avaliação com 12 indicadores. A aplicabilidade da proposta foi testada em 15 municípios da macrorre- gião da Grande Florianópolis, Santa Catarina, Brasil. O modelo demonstrou viabilidade em municípios de diferentes portes populacionais e estruturas administrativas. Os dados foram obtidos nos sistemas locais de informação e em documentos oficiais das Secretarias de Saúde. A validade do modelo pode ser inferida pelos resultados da aplicação que foram compatíveis com a literatura.

Recursos Humanos em Saúde; Gestão em Saúde; Serviços de Saúde 


\section{Colaboradores}

S. V. Scalco, J. T. Lacerda e M. C. M. Calvo participaram da concepção, projeto, análise e interpretação dos dados e, redigiram o artigo.

\section{Referências}

1. Ministério da Saúde/Conselho Nacional de Saúde Princípios e diretrizes para a gestão do trabalho no SUS (NOB/RH-SUS). 3a Ed. Brasília: Ministério da Saúde; 2005.

2. Mendes EV. A atenção primária à saúde no SUS. Fortaleza: Escola de Saúde Pública do Ceará; 2002.

3. Borrelli FRG. A rotatividade dos profissionais de saúde na zona rural de Sergipe: um problema a ser enfrentado [Dissertação de Mestrado]. Rio de Janeiro: Escola Nacional de Saúde Pública, Fundação Oswaldo Cruz; 2004.

4. Campos CVA, Malik AM. Satisfação no trabalho e rotatividade dos médicos do Programa Saúde da Família. Rev Adm Pública 2008; 42:347-68.

5. Anselmi ML, Angerami ELS, Gomes ELR. Rotatividade dos trabalhadores de enfermagem nos hospitais do Município de Ribeirão Preto. Rev Panam Salud Pública 1997; 2:44-50.

6. Arias EHL, Vitalino HA, Machado MH, Filho WA Cruz LAM. Gestão do trabalho no SUS. Cad RH Saúde 2006; 3:119-34.
7. Machado MH, Aguiar Filho W, Zamberlan FX, Ribeiro SB, Mendonça EP. A mesa de negociação como instrumento para a gestão do trabalho no SUS. Cad RH Saúde 2006; 3:141-8.

8. Departamento de Gestão da Educação na Saúde, Secretaria de Gestão do Trabalho e da Educação na Saúde, Ministério da Saúde. Política de educação e desenvolvimento para o SUS: caminhos para a educação permanente em saúde: pólos de educação permanente em saúde. Brasília: Ministério da Saúde; 2004.

9. Hoefel MGD, Elizabete CS, Jandira M. A atenção à saúde do trabalhador no SUS: a proposta de constituição da RENAST. In: 3o CNST: Trabalhar Sim! Adoecer Não! Brasília: Ministério da Saúde/Ministério da Previdência Social/Ministério do Trabalho e Emprego; 2005. p. 72-8.

10. Contandriopoulos AP. Avaliando a institucionalização da avaliação. Ciênc Saúde Coletiva 2006; 11:705-11. 
11. Calvo MCM, Henrique F. Avaliação - algumas concepções teóricas sobre o tema. In: Lacerda JT, Traebert JL, organizadores. A odontologia e a estratégia saúde da família. Tubarão: Editora Unisul; 2006. p. 115-39.

12. Silva AAC, Freitas CAS. Avaliação de programas públicos no Brasil. O papel do Tribunal de Contas da União. In: Prêmio Serzedello Corrêa 2003: monografias vencedoras. Brasília: Tribunal de Contas da União/Instituto Serzedello Corrêa; 2005. p. 14-43.

13. Dessler G. Administração de recursos humanos. São Paulo: Prentice Hall; 2003.

14. Silva LMV, Formigli VL. Avaliação em saúde: limites e perspectivas. Cad Saúde Pública 1994; 10:80-91.

15. Felisberto I. Da teoria à formulação de uma Política Nacional de Avaliação em Saúde: reabrindo o debate. Ciênc Saúde Coletiva 2006; 11:554-7.

16. Novaes HMD. Avaliação de programas, serviços e tecnologias em saúde. Rev Saúde Pública 2000; 34:547-59.

17. Arias EHL, Machado MH, Sanches DR, Tomaschescki J, Santos JR. Situação dos planos de carreiras das secretarias estaduais e municipais de saúde. Cad RH Saúde 2006; 3:149-61.

18. Varella TC, Dal Poz MR, França T. Informação como recurso estratégico para a gerência de recursos humanos. In: Santana JP, Castro JL, organizadores. Capacitação em desenvolvimento de recursos humanos de saúde - CADRHU. Natal: EDUFRN; 1999. p. 151-60.
19. Pierantoni CR, Varella TC, Santos MR, França T, Garcia AC. Gestão do trabalho e da educação em saúde: recursos humanos em duas décadas do SUS. Physis (Rio J.) 2008; 18:685-704.

20. Chiavenato I. Gestão de pessoas: o novo papel dos recursos humanos nas organizações. Rio de Janeiro: Editora Campus; 1999.

21. Ministério da Saúde. Portaria GM/MS no. 1.996, de 20 de agosto de 2007. Dispõe sobre as diretrizes para a implementação da Política Nacional de Educação Permanente em Saúde e dá outras providências. Diário Oficial da União 2007; 22 ago.

22. Paula CR. Condições de trabalho, atividade e referências a agravos: um estudo em servidores públicos federais de Santa Catarina [Dissertação de Mestrado]. Florianópolis: Universidade Federal de Santa Catarina; 2006.

23. Cunha JCCB. Adoecimento e afastamento do trabalho de servidores públicos estaduais de Santa Catarina, 1995 a 2005 [Dissertação de Mestrado]. Florianópolis: Universidade Federal de Santa Catarina; 2007.

24. Ministério da Saúde/Conselho Nacional de Saúde. A prática do controle social e os conselhos de saúde em 14 reflexões. In: Ministério da Saúde, organizador. Gestão municipal de saúde - textos básicos. Rio de Janeiro: Ministério da Saúde; 2001. p. 53-78.

Recebido em 17/Nov/2008

Versão final reapresentada em 15/Dez/2009

Aprovado em 15/Jan/2010 